\title{
Vigabatrin-Induced Peripheral Visual Field Defects in Patients With Refractory Partial Epilepsy
}

\author{
Robert C. Sergott \\ Thomas Jefferson University \\ Richard M. Bittman \\ Bittman Biostat, Inc. \\ Erin M. Christen \\ AYW Consulting \\ Stephen M. Sagar \\ Lundbeck Inc. (formerly Ovation Pharmaceuticals, Inc.) \\ Follow this and additional works at: https://jdc.jefferson.edu/willsfp \\ Part of the Ophthalmology Commons \\ Let us know how access to this document benefits you
}

\section{Recommended Citation}

Sergott, Robert C.; Bittman, Richard M.; Christen, Erin M.; and Sagar, Stephen M., "VigabatrinInduced Peripheral Visual Field Defects in Patients With Refractory Partial Epilepsy" (2010). Wills Eye Hospital Papers. Paper 9.

https://jdc.jefferson.edu/willsfp/9

This Article is brought to you for free and open access by the Jefferson Digital Commons. The Jefferson Digital Commons is a service of Thomas Jefferson University's Center for Teaching and Learning (CTL). The Commons is a showcase for Jefferson books and journals, peer-reviewed scholarly publications, unique historical collections from the University archives, and teaching tools. The Jefferson Digital Commons allows researchers and interested readers anywhere in the world to learn about and keep up to date with Jefferson scholarship. This article has been accepted for inclusion in Wills Eye Hospital Papers by an authorized administrator of the Jefferson Digital Commons. For more information, please contact: JeffersonDigitalCommons@jefferson.edu. 


\section{As submitted to:}

\section{Epilepsy Research}

\section{And later published as:}

\section{Vigabatrin-Induced Peripheral Visual Field Defects in}

\section{Patients With Refractory Partial Epilepsy}

\section{Volume 92, Issue 2-3, December 2010, Pages 170-176 DOI: 10.1016/j.eplepsyres.2010.09.004}

Robert C. Sergott, ${ }^{a^{*}}$ Richard M. Bittman, ${ }^{b}$ Erin M. Christen, ${ }^{c}$ Stephen M. Sagar ${ }^{\mathrm{d}}$

${ }^{a *}$ Wills Eye Institute, Neuro-Ophthalmology Service, Thomas Jefferson University Medical College, 840 Walnut Street, Suite 930, Philadelphia, PA 19107 USA; fax: 1-215-592-1923;

e-mail: rcs220@comcast.net

${ }^{\mathrm{b}}$ Bittman Biostat, Inc., Glencoe, Illinois, USA; e-mail: rmb@bittmanbiostat.com ${ }^{\mathrm{c}}$ AYW Consulting, Knoxville, Tennessee, USA; e-mail: aywconsulting@gmail.com ${ }^{\mathrm{d}}$ Lundbeck Inc. (formerly Ovation Pharmaceuticals, Inc.), Deerfield, Illinois, USA; e-mail: SSAG@lundbeck.com

Running title: Vigabatrin-Induced Peripheral Visual Field Defects 
Keywords: Vigabatrin, Retinopathy, Visual Field Constriction, Epilepsy, Clinical Trial

*Corresponding author 


\section{SUMMARY}

Purpose: Vigabatrin can cause retinopathy, resulting in bilateral visual field constriction.

Previous analyses of results from a prospective, observational study assessing vigabatrin-induced visual field constriction (described below) employed a partially subjective interpretation of static perimetery. In an effort to affirm these previous findings through more objective, quantitative methodology, we now report data from a subset analysis of refractory partial epilepsy patients in the study who underwent Goldmann kinetic perimetry.

Methods: Patients aged $\geq 8$ years with refractory partial seizures were enrolled and grouped: those receiving vigabatrin for $\geq 6$ months (Group I); those who had received vigabatrin for $\geq 6$ months and then had discontinued for $\geq 6$ months (Group II); and those naïve to vigabatrin (Group III). Patients underwent static or kinetic perimetry or both every 4-6 months for $\leq 3$ years. For kinetic perimetry, the temporal and nasal visual fields were measured along the horizontal meridian with the largest (V4e, IV4e) and smallest (I2e, I1e) isopters, respectively. Results: Of 735 patients enrolled, 341 had Goldmann perimetry data. Of these, 258 received vigabatrin. Sixteen percent of vigabatrin-exposed patients had moderate visual field defects $\left(30^{\circ}-60^{\circ}\right.$ retained temporal vision), and $3 \%$ had severe defects $\left(<30^{\circ}\right.$ retained temporal vision). Visual function questionnaire results indicated a weak correlation between visual field constriction severity and visual symptoms.

Conclusions: These results affirm both an analysis of the same study based primarily on static perimetry and findings from cross-sectional studies. The present analysis verifies that visual field constriction, when it occurs, is most often mild or moderate and is not associated with symptoms 
of abnormal visual function. The clinical decision to prescribe vigabatrin should be based on a benefit-risk analysis for each individual patient. 


\section{INTRODUCTION}

Vigabatrin $\left(\right.$ Sabril $\left.^{\circledR}\right)$, a selective and irreversible $\gamma$-aminobutyric acid transaminase inhibitor (Ben-Menachem, 1995), is effective as adjunctive therapy for adult patients with refractory complex partial seizures (Bruni et al., 2000, French et al., 1996, Guberman and Bruni, 2000, Sander et al., 1990) and as monotherapy for infants with infantile spasms (West syndrome) (Aicardi et al., 1996, Appleton et al., 1999, Chiron et al., 1990, Chiron et al., 1997, Elterman et al., 2001, Wohlrab et al., 1998). Vigabatrin has been approved for these indications in the United States since 2009, and in many other countries since 1989. Despite its proven efficacy, the use of vigabatrin has been limited by the associated risk of retinopathy, characterized by irreversible, bilateral constriction of the visual fields, an adverse reaction to the drug first reported in 1997 (Eke et al., 1997). Since then, several reports have described this very specific visual field defect in vigabatrin-treated patients (Baulac et al., 1998, Harding, 1998, Kalviainen and Nousiainen, 2001, Lawden et al., 1999, Nicolson et al., 2002, Wild et al., 1999, Wilson and Brodie, 1997), and systematic retrospective (Besch et al., 2002, Hardus et al., 2000, Kalviainen and Nousiainen, 2001, Kalviainen et al., 1999, Newman et al., 2002, Nicolson et al., 2002, Stefan et al., 2000, Vanhatalo et al., 2002, Wild et al., 1999) and prospective (Schmitz et al., 2002) analyses have suggested that it may occur in a significant number of treated patients (reported range, 14\%92\%). This large range is influenced by differing visual field testing methods, as well as the criteria defining abnormal fields. The visual field defect generally is asymptomatic (Wild et al., 1999), possibly a result of, in part, the relative sparing of the central retina. However, there is electrophysiologic evidence, using multifocal electroretinography, that vigabatrin causes a reduction in function within the central retina. Other studies have reported mild decreases in 
color vision (Johnson et al., 2000) and reductions in grating acuity in children (Durbin et al., 2009).

In response to concerns of the European Medicines Agency, Sanofi-Aventis, the manufacturer of vigabatrin outside of North America, conducted a large cross-sectional study (Study 4020) with a prospective follow-up to further investigate vigabatrin-induced visual field constriction in pediatric and adult patients being treated for partial epilepsy. The study was designed to assess the frequency of the vigabatrin-induced visual field constriction, better characterize the functional consequences of the visual defect, and determine risk factors for its development. An interim analysis of this study and a partial analysis of the final data have been published. In these analyses, the primary outcome measure, the presence or absence of bilateral, concentric, peripheral constriction (BCPC) of the visual fields, was based on a global assessment of the perimetry data for each patient through partially specified criteria and predominantly static perimetry. The analysis of the final data set found that, among 524 evaluable patients with 1 or more conclusive perimetry examinations, the frequency of $\mathrm{BCPC}$ at the last conclusive examination was greatest for patients receiving vigabatrin at study entry: $26.3 \%$ for patients aged 8-12 years and $43.3 \%$ for patients aged $>12$ years (Wild et al., 2009). For patients who had discontinued vigabatrin for $\geq 6$ months, the frequencies were $14.9 \%$ for children and $24.5 \%$ for adults. Further, this report found that the risk of developing BCPC was correlated significantly with duration of vigabatrin therapy, mean daily dosage, and male sex. Among 386 vigabatrinexposed patients, visual field loss was graded mild for $15.8 \%$, moderate for $10.6 \%$, and severe for $3.9 \%$, based on a complex grading system applied to data from both static and kinetic perimetry. Moreover, no relationship was found between the presence of symptoms of visual 
difficulties ascertained by questionnaire and vigabatrin-induced visual field constriction (Wild et al., 2009).

While for many patients, static perimetry has very low test/retest variability, for other patients, the rigor of the testing procedures, combined with possible fatigue and decreased cognitive function, produces high coefficients of variability. Because of the subjective nature of static perimetery for some patients, we sought to verify the findings of the previous research by conducting objective and quantitative analyses to affirm the rates and severity of vigabatrininduced visual field constriction previously observed. Through the present analyses, we provide the results for a subset of 341 patients in Study 4020 who underwent Goldmann kinetic perimetry. 


\section{METHODS}

\subsection{Study Design}

This was an open-label, multicenter, comparative study conducted at 46 sites in France, South Korea, Italy, Spain, and Australia. Male and female patients $\geq 8$ years of age with refractory partial seizures for $\geq 1$ year were eligible. Patients were classified into 6 groups defined by 2 age groups and 3 treatment groups (Table 1). Children were 8-12 years of age, and adults were $>12$ years. Group I was composed of patients who were receiving vigabatrin at study entry and who had been treated with vigabatrin as either monotherapy or polytherapy for $\geq 6$ months before study entry. Group II included patients who were treated with other antiepileptic drugs (AEDs) for $\geq 6$ months but who had previously received vigabatrin for $\geq 6$ months and had discontinued vigabatrin $\geq 6$ months prior to study entry. Patients in Group III were treated with other AEDs and had never received vigabatrin. However, these patients were permitted to initiate treatment with vigabatrin during the study. Patients with identified primary ophthalmologic pathology were excluded. All patients, or their caregivers, provided written informed consent.

This study consisted of an initial screening/randomization phase, baseline visit, and a follow-up phase of up to 36 months. The baseline ophthalmologic assessments included ocular history and symptoms, listing of all ocular medications taken during the past 6 months, eye color, visual acuity, manifest refraction, slit-lamp biomicroscopy, intraocular pressure, bilateral dilated ophthalmoscopy, gonioscopy, perimetry, determination of whether any ocular findings might explain abnormal visual field results, and visual disability as assessed by a questionnaire. Perimetry (static, kinetic, or both) was repeated every 4-6 months at each follow-up visit. Sites were allowed to choose the perimetry device and protocol employed within broad parameters. A 
site did not necessarily employ a consistent methodology, even for different visits of an individual patient.

A subset of patients underwent kinetic perimetry testing — the vast majority via the Goldmann perimeter and the balance through the Moniteur device. The data from Goldmann perimetry were subjected to post-hoc analysis. Digital files of scanned perimetry records from these tests were submitted to an independent contract research organization (ORA Clinical Research and Development, Andover, MA, USA), which measured the extent of the visual fields along the horizontal meridian while blinded to treatment group. Measurements were conducted for the largest isopter tested in the temporal field and the smallest isopter tested in the nasal field. The majority of the data available were from the V4e and IV4e temporal isopters and the I2e and I1e nasal isopters. Because of non-uniformity between or within sites regarding the isopters tested, the data are presented in 2 ways. First, the measurements for the largest temporal and smallest nasal isopters tested were combined to generate summary data representing all the observations. Second, as a way of guarding against misleading results from combining disparate measurements, the measurements were analyzed separately for the 2 temporal and 2 nasal isopters with the most data.

At each study visit, patients completed a 19-item visual function questionnaire, primarily consisting of questions concerning peripheral vision (eg, do you bump into doors, do you have trouble catching a ball). 


\subsection{Treatment}

The dosage and regimen of vigabatrin were not protocol-determined, with the exception of 7 patients in Group III who were prescribed vigabatrin after enrollment. For these patients, the

daily starting dosages were $1 \mathrm{~g} / \mathrm{day}$ for adults and $40 \mathrm{mg} / \mathrm{kg}$ for children, and these dosages could be titrated up to $2-3 \mathrm{~g} /$ day for adults and $80-100 \mathrm{mg} / \mathrm{kg}$ for children. All other patients were allowed to continue the AED treatment they were receiving at study entry, with dosage adjustments as deemed necessary by their physicians.

\subsection{Data Analysis}

Measurements employed in the analyses of the largest temporal and smallest nasal isopters were from the last perimetry obtained for each patient during the study. For an individual isopter, the data were from the last perimetry obtained with that isopter, which was not necessarily the final perimetry obtained during the study. 


\section{RESULTS}

\subsection{Patients}

A total of 735 patients enrolled in the study (Figure 1). Measurements of extent of visual fields based on kinetic perimetry were obtained for 359 patients. Of these 359,341 had undergone Goldmann perimetry and 18 had completed perimetry with the Moniteur device. Of the 341 patients who completed Goldmann perimetry, 258 were exposed to vigabatrin and 83 were naive to the drug. The distribution of these patients by treatment and age groups is shown in Table 1, and baseline characteristics are provided in Table 2. Distribution by sex was approximately equal in adults, but there was a male preponderance among children. Mean age at study entry was 10.3 years for children and 33.1 years for adults. At study entry, the median duration of vigabatrin treatment was 4.1 years for both children and adults in Group I, and 1.6 years for children and 2.5 years for adults in Group II. For children and adults in Groups I and II, mean time from first vigabatrin dose to first perimetry examination ranged from 4.0 to 5.7 years. Most baseline characteristics of patients in Group III were similar to those of patients treated with vigabatrin, except that patients in Group III had shorter AED exposure times than those in either Group I or Group II. Moreover, the subset of patients who had undergone kinetic perimetry generally was representative of the entire group of patients in the study. However, in this subset, the median durations of vigabatrin exposure were shorter for adults in Group I (3.5 vs. 4.6 years) and children in Group II (1.6 vs. 3.0 years).

\subsection{Perimetry}

The extent of visual field impairment was analyzed for the subset of 341 patients who had completed Goldmann perimetry. All patients had data for the temporal field, and all but 2 had 
data for the nasal field. Based on combined data for the largest isopter tested, monocular temporal field at final Goldmann perimetry for the 258 vigabatrin-exposed patients ranged from $13^{\circ}$ to $90^{\circ}$, and averaged $71.1^{\circ} \pm 14.7^{\circ}$ (Table 3 and Figure $2 \mathrm{a}$ ). When analyzed for the V4e and IV4e isopters, for which the most data were available, the mean retained temporal fields for vigabatrin-exposed patients were $73.5^{\circ} \pm 15.3^{\circ}\left(\right.$ range, $\left.12^{\circ}-90^{\circ}\right)$ and $69.1^{\circ} \pm 15.4^{\circ}\left(\right.$ range, $7^{\circ}-90^{\circ}$ ), respectively.

Monocular nasal fields of vigabatrin-exposed patients, based on the smallest isopter tested, ranged from $0^{\circ}$ to $64^{\circ}$, averaging $19.7^{\circ} \pm 10.8^{\circ}$ (Table 3 and Figure $2 \mathrm{~b}$ ). When analyzed for the I2e and I1e isopters, for which the most data were available, the mean retained nasal fields were $19.0^{\circ} \pm 8.3^{\circ}\left(\right.$ range, $4^{\circ}-37^{\circ}$ ) and $13.2^{\circ} \pm 7.1^{\circ}$ (range, $3^{\circ}-36^{\circ}$ ), respectively

The perimetry results from vigabatrin-exposed and vigabatrin-naïve patients exhibited modest differences. Depending on the isopters compared, the mean retained temporal field was between $6.9^{\circ}$ and $8.9^{\circ}$ greater for vigabatrin-naïve patients, while the difference in mean retained nasal field varied between $1.8^{\circ}$ and $2.4^{\circ}$ (Table 3). Cumulative frequency distribution of retained visual field from the combined isopter data demonstrates modest differences between the vigabatrin-exposed and vigabatrin-naïve patients (Figure 2).

The retained temporal fields were classified as normal $\left(>80^{\circ}\right.$ retained field, averaged for the 2 eyes $)$, mildly impaired $\left(60^{\circ}-80^{\circ}\right)$, moderately impaired $\left(30^{\circ}-<60^{\circ}\right)$, and severely impaired $\left(<30^{\circ}\right)$. The distributions of temporal visual field impairment at the last kinetic perimetry examination for the largest isopter tested for vigabatrin-exposed and vigabatrin-naïve patients are 
shown in Figures 3a and 3b, respectively. Of vigabatrin-exposed patients, the majority were unimpaired or had mild or moderate impairment of temporal visual fields, while $2 \%$ had severe impairment. Another approach employed data for vigabatrin-naïve patients to calculate the retained visual field measurements representing the fifth, tenth, and twenty-fifth percentiles and applied those percentile ranges to the vigabatrin-exposed patients. By these criteria, for the temporal field results of the largest isopters tested, $20.2 \%$ of vigabatrin-exposed patients were within the fifth percentile $\left(61.5^{\circ}\right), 28.7 \%$ were within the tenth percentile $\left(65.5^{\circ}\right)$, and $41.5 \%$ were within the twenty-fifth percentile $\left(71.5^{\circ}\right)$

\subsection{Visual Symptoms}

Results from the questionnaire, which assessed visual symptoms, such as bumping into objects and having difficulty catching a ball, showed only a weak correlation between perimetry results and symptoms of visual disability. When patients who had completed Goldmann perimetry were stratified according to the severity of visual field defect, as described in Section 3.2, we found a weak ( $r=0.16)$ but statistically significant $(P=0.005)$ correlation between severity of visual field defect and answering "yes" to at least 1 item of the 19-item questionnaire (data not shown). 


\section{DISCUSSION}

The original study sought to estimate the prevalence and incidence of visual field defect for children and adults treated with vigabatrin for refractory partial epilepsy and to identify risk factors for their occurrence (Wild et al., 2009). It included the largest group of vigabatrinexposed epilepsy patients ever systematically evaluated for vision loss. The primary outcome measure was a qualitative determination of bilateral, concentric, peripheral constriction (BCPC) by a single visual field expert who was blinded to treatment group, but who had access to all perimetry data for each patient. Some patients underwent both static and kinetic perimetry at the same visit, and for those patients, the reviewer placed greater weight on static perimetry (Wild et al., 2009). The criteria used for determining BCPC have been described previously (Wild et al., 1999). The advantages of this qualitative approach include consistency and the ability to incorporate all available perimetry data for each patient, while the disadvantages of the approach are a lack of objective, protocol-specified BCPC criteria and potential bias.

In addition to the outcome measurement, this analysis had several limitations. First, the study included only 7 patients who had initiated vigabatrin therapy during the study (6 of whom had Goldmann perimetry and none of whom developed visual field constriction). Second, baseline data were not available for any of the remaining patients. Third, given that $\geq 6$ months of vigabatrin exposure was required for Groups I and II and the mean exposure at enrollment was actually between 1.6 and 4.1 years for all groups, the earliest onset of visual field constriction during vigabatrin therapy could not be assessed in this study. Instead, cases of vigabatrininduced visual field constriction were detected only after several months or years of vigabatrin exposure. By contrast, a well-characterized case of visual field constriction has been reported 
after only 4 months of vigabatrin exposure (Malmgren et al., 2001). The literature contains a dearth of reports of visual field testing for patients with shorter durations of drug exposure. Fourth, the study lacked a uniform testing method between the 46 sites or even within a single site. Finally, the study collected vigabatrin exposure data retrospectively. However, despite its limitations, this study was the largest to assess vision effects in vigabatrin-treated patients, providing an estimate of the frequency and severity of visual field constriction with durations of vigabatrin exposure between 6 months and approximately 5 years.

The current paper complements the previously published analysis of this same study (Wild et al., 2009). This analysis employed quantitative measurements of the visual field along the horizontal meridian from kinetic perimetry to estimate the severity of visual field defects. While this quantitative approach was limited by the lack of uniformity in perimetry methodology (in particular the failure to test the same isopters for all patients at all visits), we mitigated that limitation by analyzing data from individual isopters to support the findings and to summarize the entire data set with descriptive statistics for combined measurements of several isopters.

No generally agreed upon categorization of the severity of visual field defect exists. The severity categories chosen for the current analysis were intended to reflect the degree of resulting functional limitation. For example, $120^{\circ}$ of total width of the horizontal visual field (equivalent to $60^{\circ}$ of monocular temporal vision) is the limit for having a driver's license in many jurisdictions and was chosen as the dividing line between mild and moderate defect. The criterion of $<30^{\circ}$ for a severe deficit is similar to that chosen for several published studies based on kinetic perimetry. Importantly, the categories employed here are not commensurate with those 
employed by Wild et al (Wild et al., 2009). Moreover, the duration of vigabatrin exposure was somewhat shorter in the subgroup of patients undergoing Goldmann perimetry than in the entire study population.

The quantitative assessment of kinetic perimetries (Figure 3) demonstrated that the majority of vigabatrin-exposed patients had some constriction of the visual fields. However, this should be compared with the finding that $45 \%$ of vigabatrin-naïve patients also had retained temporal visual field $<80^{\circ}$. The majority of vigabatrin-induced visual field defects were mild to moderate (Figure 3). Nevertheless, severe visual field constriction to $<30^{\circ}$ of retained monocular temporal visual field, corresponding to a total extent of binocular vision of $<60^{\circ}$, occurred in $2 \%$ of patients. In spite of the differences between the analyses, Wild and colleagues (Wild et al., 2009) found that 15 of 386 patients (4\%) in Groups I and II had severe vigabatrin-attributed visual field loss, which is similar to the findings of our present analysis. However, in contrast to the analysis of Wild and colleagues (Wild et al., 2009), we found a weak correlation between answering "yes" to at least 1 question on the visual function questionnaire and severity of the visual field defect.

In the assessment of patients receiving vigabatrin, this analysis does not demonstrate that kinetic perimetry is necessarily superior to static perimetry. It is the clinician's decision which method should be used for an individual patient. For some patients, (for example, those who are lethargic or have attention deficits), kinetic perimetry may be preferable, as it provides for more humanto-human interaction and monitoring. On the other hand, for those are alert, cognitive intact, and 
engaged, static perimetry may be appropriate. One size does not fit all, and judgment on patientby-patient basis is warranted.

In conclusion, this analysis of data from the largest cross-sectional study conducted to date of perimetry in patients treated with vigabatrin for refractory partial epilepsy verifies that visual field constriction, when it occurs, is most often mild or moderate and is not associated with symptoms of abnormal visual function. However, for a minority of patients exposed to vigabatrin for months to years, the visual field constriction can be severe and symptomatic.

The clinical decision to prescribe vigabatrin should be based on a benefit-risk analysis for each individual patient. Peripheral visual field constriction caused by vigabatrin is asymptomatic for most patients, and, thus, periodic ophthalmologic testing (including perimetry) is required to detect the defect. For patients with refractory complex partial epilepsy who do not experience a substantial improvement in seizure control after 1- to 3-month trials of vigabatrin, the drug should be discontinued to minimize the risk of vision loss. For patients who receive substantial benefit from vigabatrin, periodic ophthalmologic testing is recommended to help guide physicians in their ongoing benefit-risk analyses for all patients (Sergott et al., 2010). 


\section{ACKNOWLEDGMENTS}

The authors acknowledge and thank Dr. Randy Kardon, University of Iowa, for his advice and recommendations with respect to the data analyses reported in this manuscript. We also thank Marithea Goberville, $\mathrm{PhD}$, and Angela Fracasso, of R\&R Healthcare Communications, and Michael A. Nissen, ELS, of Lundbeck Inc., for editorial assistance in the development and revision of this manuscript. This support was funded by Lundbeck.

\section{DISCLOSURES}

The authors had complete access to all study data and take full responsibility for the integrity of the data, the accuracy of the analysis, and the opinions expressed in the manuscript. Each author was involved in the design, analysis, and interpretation of the data. All authors approved the final version of the manuscript and granted permission for its submission for publication.

RCS, RMB, and EMC have served as paid consultants to Lundbeck Inc. SMS is a full-time employee of Lundbeck, Inc.

\section{FUNDING}

The original study reported in this manuscript was conducted by Sanofi-Aventis. Copies of the visual field recordings were transferred to Lundbeck Inc. (formerly Ovation Pharmaceuticals), who acquired the US rights to vigabatrin from Aventis. The visual field recordings were measured by a contract research organization (CRO) funded by Lundbeck. 
Lundbeck financially supported this analysis, and a Lundbeck author (SMS) participated in the data analysis reported here, as well as reviewed and approved the manuscript. Lundbeck granted approval for the submission as well. 


\section{REFERENCES}

Aicardi, J., Mumford, J.P., Dumas, C., Wood, S., 1996. Vigabatrin as initial therapy for infantile spasms: a European retrospective survey. Sabril IS Investigator and Peer Review Groups. Epilepsia 37, 638-42.

Appleton, R.E., Peters, A.C., Mumford, J.P., Shaw, D.E., 1999. Randomised, placebo-controlled study of vigabatrin as first-line treatment of infantile spasms. Epilepsia 40, 1627-33.

Baulac, M., Nordmann, J.P., Lanoe, Y., 1998. Severe visual-field constriction and side-effects of GABA-mimetic antiepileptic agents. Lancet 352, 546.

Ben-Menachem, E., 1995. Vigabatrin. Epilepsia 36 Suppl 2, S95-104.

Besch, D., Kurtenbach, A., Apfelstedt-Sylla, E., Sadowski, B., Dennig, D., Asenbauer, C., et al., 2002. Visual field constriction and electrophysiological changes associated with vigabatrin. Doc Ophthalmol 104, 151-70.

Bruni, J., Guberman, A., Vachon, L., Desforges, C., 2000. Vigabatrin as add-on therapy for adult complex partial seizures: a double-blind, placebo-controlled multicentre study. The Canadian Vigabatrin Study Group. Seizure 9, 224-32.

Chiron, C., Dulac, O., Luna, D., Palacios, L., Mondragon, S., Beaumont, D., et al., 1990. Vigabatrin in infantile spasms. Lancet 335, 363-4.

Chiron, C., Dumas, C., Jambaque, I., Mumford, J., Dulac, O., 1997. Randomized trial comparing vigabatrin and hydrocortisone in infantile spasms due to tuberous sclerosis. Epilepsy Res 26, $389-95$. 
Durbin, S., Mirabella, G., Buncic, J.R., Westall, C.A., 2009. Reduced grating acuity associated with retinal toxicity in children with infantile spasms on vigabatrin therapy. Invest Ophthalmol Vis Sci 50, 4011-6.

Eke, T., Talbot, J.F., Lawden, M.C., 1997. Severe persistent visual field constriction associated with vigabatrin. BMJ 314, 180-1.

Elterman, R.D., Shields, W.D., Mansfield, K.A., Nakagawa, J., 2001. Randomized trial of vigabatrin in patients with infantile spasms. Neurology 57, 1416-21.

French, J.A., Mosier, M., Walker, S., Sommerville, K., Sussman, N., 1996. A double-blind, placebo-controlled study of vigabatrin three $\mathrm{g} /$ day in patients with uncontrolled complex partial seizures. Vigabatrin Protocol 024 Investigative Cohort. Neurology 46, 54-61.

Guberman, A., Bruni, J., 2000. Long-term open multicentre, add-on trial of vigabatrin in adult resistant partial epilepsy. The Canadian Vigabatrin Study Group. Seizure 9, 112-8.

Harding, G.F., 1998. Severe persistent visual field constriction associated with vigabatrin. Benefit: risk ratio must be calculated for individual patients. BMJ 316, 232-3.

Hardus, P., Verduin, W.M., Postma, G., Stilma, J.S., Berendschot, T.T., Van Veelen, C.W., 2000. Concentric contraction of the visual field in patients with temporal lobe epilepsy and its association with the use of vigabatrin medication. Epilepsia 41, 581-7.

Johnson, M.A., Krauss, G.L., Miller, N.R., Medura, M., Paul, S.R., 2000. Visual function loss from vigabatrin: effect of stopping the drug. Neurology 55, 40-5.

Kalviainen, R., Nousiainen, I., 2001. Visual field defects with vigabatrin: epidemiology and therapeutic implications. CNS Drugs 15, 217-30. 
Kalviainen, R., Nousiainen, I., Mantyjarvi, M., Nikoskelainen, E., Partanen, J., Partanen, K., et al., 1999. Vigabatrin, a gabaergic antiepileptic drug, causes concentric visual field defects. Neurology 53, 922-6.

Lawden, M.C., Eke, T., Degg, C., Harding, G.F., Wild, J.M., 1999. Visual field defects associated with vigabatrin therapy. J Neurol Neurosurg Psychiatry 67, 716-22.

Malmgren, K., Ben-Menachem, E., Frisen, L., 2001. Vigabatrin visual toxicity: evolution and dose dependence. Epilepsia 42, 609-15.

Newman, W.D., Tocher, K., Acheson, J.F., 2002. Vigabatrin associated visual field loss: a clinical audit to study prevalence, drug history and effects of drug withdrawal. Eye 16, 567-71.

Nicolson, A., Leach, J.P., Chadwick, D.W., Smith, D.F., 2002. The legacy of vigabatrin in a regional epilepsy clinic. J Neurol Neurosurg Psychiatry 73, 327-9.

Sander, J.W., Trevisol-Bittencourt, P.C., Hart, Y.M., Shorvon, S.D., 1990. Evaluation of vigabatrin as an add-on drug in the management of severe epilepsy. J Neurol Neurosurg Psychiatry 53, 1008-10.

Schmitz, B., Schmidt, T., Jokiel, B., Pfeiffer, S., Tiel-Wilck, K., Ruther, K., 2002. Visual field constriction in epilepsy patients treated with vigabatrin and other antiepileptic drugs: a prospective study. J Neurol 249, 469-75.

Sergott, R.C., Wheless, J.W., Smith, M.C., Westall, C.A., Kardon, R.H., Arnold, A., et al., 2010. Evidence-based review of recommendations for visual function testing in patients treated with vigabatrin. Neuro-ophthalmology 34, 20-35.

Stefan, H.J., Bernati, K., Knorr, H.L.J., 2000. Visual field constriction and anti-epileptic drug treatment. Neurol Psychiat Brain Research 7, 185-190. 
Vanhatalo, S., Nousiainen, I., Eriksson, K., Rantala, H., Vainionpaa, L., Mustonen, K., et al., 2002. Visual field constriction in 91 Finnish children treated with vigabatrin. Epilepsia 43, 74856.

Wild, J.M., Chiron, C., Ahn, H., Baulac, M., Bursztyn, J., Gandolfo, E., et al., 2009. Visual field loss in patients with refractory partial epilepsy treated with vigabatrin: final results from an openlabel, observational, multicentre study. CNS Drugs 23, 965-82.

Wild, J.M., Martinez, C., Reinshagen, G., Harding, G.F., 1999. Characteristics of a unique visual field defect attributed to vigabatrin. Epilepsia 40, 1784-94.

Wilson, E.A., Brodie, M.J., 1997. Severe persistent visual field constriction associated with vigabatrin. Chronic refractory epilepsy may have role in causing these unusual lesions. BMJ 314, 1693.

Wohlrab, G., Boltshauser, E., Schmitt, B., 1998. Vigabatrin as a first-line drug in West syndrome: clinical and electroencephalographic outcome. Neuropediatrics 29, 133-6. 


\section{TABLES}

Table 1. Study group based on age and treatment history (patients with Goldmann perimetry data)

\begin{tabular}{|l|l|c|c|}
\hline \multicolumn{2}{|c|}{} & \multicolumn{2}{|c|}{ Patients, n } \\
\cline { 2 - 4 } Group & $\begin{array}{c}\text { Children } \\
\text { (8-12 years })\end{array}$ & $\begin{array}{c}\text { Adults } \\
\text { (>12 years })\end{array}$ \\
\hline I & $\begin{array}{l}\text { Patients currently being treated with } \\
\text { vigabatrin for } \geq 6 \text { months }\end{array}$ & 28 & 92 \\
\hline II & $\begin{array}{l}\text { Patients previously treated with } \\
\text { vigabatrin for } \geq 6 \text { months who had } \\
\text { discontinued for } \geq 6 \text { months before } \\
\text { study entry }\end{array}$ & 32 & 100 \\
\hline III & $\begin{array}{l}\text { Patients never treated with vigabatrin } \\
\text { before study entry }\end{array}$ & 22 & 67 \\
\hline
\end{tabular}

*6 patients in Group III began receiving vigabatrin during the course of the study and are included in the data analyses for both the vigabatrin-exposed and vigabatrin-naïve groups. 
Table 2. Baseline characteristics of the study groups

\begin{tabular}{|c|c|c|c|c|c|c|}
\hline \multirow[b]{2}{*}{ Characteristic } & \multicolumn{3}{|c|}{ Children $(\mathrm{n}=82)$} & \multicolumn{3}{|c|}{ Adults $(n=259)$} \\
\hline & $\begin{array}{c}\text { Group I } \\
(n=28)\end{array}$ & $\begin{array}{l}\text { Group II } \\
(\mathbf{n}=32)\end{array}$ & $\begin{array}{l}\text { Group III } \\
(\mathrm{n}=22)\end{array}$ & $\begin{array}{l}\text { Group I } \\
(\mathbf{n}=92)\end{array}$ & $\underset{(n=100)}{\operatorname{Group} I I}$ & $\begin{array}{c}\text { Group III } \\
(n=67)\end{array}$ \\
\hline Sex, n $(\%)$ & & & & & & \\
\hline Male & $14(50.0)$ & $20(62.5)$ & $14(63.6)$ & $40(43.5)$ & $54(54.0)$ & $29(43.3)$ \\
\hline Female & $14(50.0)$ & $12(37.5)$ & $8(36.4)$ & $52(56.5)$ & $46(46.0)$ & $38(56.7)$ \\
\hline Mean age, years \pm SD & $10.3 \pm 1.4$ & $10.1 \pm 1.3$ & $10.5 \pm 1.3$ & $33.0 \pm 15.3$ & $33.2 \pm 14.5$ & $33.0 \pm 14.3$ \\
\hline $\begin{array}{l}\text { Mean duration of vigabatrin } \\
\text { dosing at study entry, years } \\
\pm \text { SD }\end{array}$ & $4.1 \pm 2.8$ & $1.6 \pm 1.7$ & NA & $4.1 \pm 2.6$ & $2.5 \pm 2.2$ & NA \\
\hline $\begin{array}{l}\text { Mean time from first } \\
\text { vigabatrin dose to first } \\
\text { perimetry examination, } \\
\text { years } \pm \text { SD }\end{array}$ & $\begin{array}{l}4.1 \pm 2.8 \\
(n=28)\end{array}$ & $\begin{array}{l}4.0 \pm 2.5 \\
(n=31)\end{array}$ & NA & $\begin{array}{l}4.1 \pm 2.6 \\
(n=92)\end{array}$ & $\begin{array}{l}5.7 \pm 2.8 \\
(n=100)\end{array}$ & NA \\
\hline $\begin{array}{l}\text { Mean duration of AED use, } \\
\text { years } \pm \text { SD }\end{array}$ & $\begin{array}{l}4.4 \pm 3.0 \\
(n=21)\end{array}$ & $\begin{array}{l}4.1 \pm 3.0 \\
(n=32)\end{array}$ & $\begin{array}{c}3.3 \pm 1.9 \\
(\mathrm{n}=22)\end{array}$ & $\begin{array}{l}9.6 \pm 7.6 \\
(n=83)\end{array}$ & $\begin{array}{l}8.8 \pm 7.5 \\
(n=97)\end{array}$ & $\begin{array}{c}10.0 \pm 10.0 \\
\quad(n=67)\end{array}$ \\
\hline
\end{tabular}

$\mathrm{AED}=$ antiepileptic drug; $\mathrm{NA}=$ not applicable; $\mathrm{SD}=$ standard deviation. 
Table 3. Degrees of retained visual field along the horizontal meridian.

\begin{tabular}{|l|l|l|c|c|c|c|c|}
\hline Group & Isopter & n & Mean & Median & Minimum & Maximum & SD \\
\hline \multicolumn{7}{|l|}{ Temporal Field } \\
\hline VGB-exposed & Largest & 258 & 71.1 & 73.0 & 13.0 & 90.0 & 14.7 \\
\hline & V4e & 135 & 73.5 & 77.0 & 11.5 & 90.0 & 15.3 \\
\hline & IV4e & 131 & 69.1 & 72.5 & 7.0 & 90.0 & 15.4 \\
\hline VGB-naïve & Largest & 83 & 78.9 & 82.0 & 27.0 & 91.0 & 12.3 \\
\hline & V4e & 40 & 82.4 & 87.3 & 25.0 & 91.0 & 13.9 \\
\hline & IV4e & 38 & 76.0 & 80.0 & 39.5 & 90.0 & 13.7 \\
\hline Nasal Field & & & & & & \\
\hline VGB-exposed & Smallest & 256 & 19.7 & 19.0 & 0.0 & 63.5 & 10.8 \\
\hline & I2e & 86 & 19.0 & 21.3 & 4.0 & 36.5 & 8.3 \\
\hline & I1e & 157 & 13.2 & 11.5 & 3.0 & 36.0 & 7.1 \\
\hline VGB-naïve & Smallest & 81 & 21.5 & 20.0 & 3.5 & 46.5 & 11.9 \\
\hline & I2e & 28 & 21.8 & 24.8 & 0.0 & 37.0 & 10.5 \\
\hline & I1e & 44 & 15.6 & 14.5 & 4.0 & 41.0 & 8.7 \\
\hline
\end{tabular}

$\mathrm{SD}=$ standard deviation; $\mathrm{VGB}=$ vigabatrin . 
FIGURE LEGENDS

Figure 1. Distribution of patients who underwent kinetic perimetry.

Figure 2. Cumulative distribution of degrees in the (A) temporal visual field at final perimetry in vigabatrin-exposed $(n=258)$ and vigabatrin-naïve patients $(n=83)$ and $(B)$ nasal visual field at final perimetry in vigabatrin-exposed $(n=256)$ and vigabatrin-naïve $(n=81)$ patients.

Figure 3. Severity of visual field defect at last kinetic perimetry in (A) vigabatrin-exposed and (B) vigabatrin-naïve patients. Unimpaired: $>80^{\circ}$ monocular temporal field retained; Mild: $60^{\circ}-80^{\circ}$ monocular temporal field retained; Moderate: $30^{\circ}-60^{\circ}$ monocular temporal field retained; Severe: $<30^{\circ}$ monocular temporal field retained. Measurements are of largest isopter tested at final Goldmann perimetry. 